\title{
Chronic Toxicity Study in Rats Orally Exposed to Mulberry Sea-Buckthorn Beverage Concentrate
}

\author{
Qingping Sun*, Zhimin Xu \\ Guangdong Institute of Food and Drug Control, Guangzhou, China \\ Email: wdsqp@hotmail.com
}

Received 28 May 2014; revised 12 July 2014; accepted 18 August 2014

Copyright (C) 2014 by authors and Scientific Research Publishing Inc. This work is licensed under the Creative Commons Attribution International License (CC BY). http://creativecommons.org/licenses/by/4.0/

(c) (i) Open Access

\section{Abstract}

Objective: This study is designed to observe the chronic toxicity after the administration of mulberry sea-buckthorn beverage concentrate for 3 months on rats and to predict the possible adverse effect and the potential toxicity target organs. Method: The rats (SPF level) were randomly divided into high-dose $(20 \mathrm{~mL} / \mathrm{kg} \mathrm{BW})$, middle-dose $(10 \mathrm{~mL} / \mathrm{kg} \mathrm{BW})$, low-dose $(5 \mathrm{~mL} / \mathrm{kg} \mathrm{BW})$ groups and negative control group $(20 \mathrm{~mL} / \mathrm{kg} B W$ of purified water) with 30 rats in each group. Each group was orally given mulberry sea-buckthorn beverage concentrate for 3 months and recovered by stop feeding samples for 2 weeks for a recovery observation. The rats' general condition, the organ coefficient, the indexes of hematology and blood biochemistry and the histological changes of the main organs were determined. Result: The appearance and behavior of activity in rats showed no anomalies in all these groups and all the rats put on weight during this period. Comparing to the negative control group, no obvious differences were observed in the weekly weight and organ coefficient of each dose group. After 3 months of administration, HGB in both mulberry sea-buckthorn beverage concentrate low-dose group and high-dose group were increased. No significant differences were observed in the indexes of hematology after 2 weeks of recovery. CREA in low-dose, middle-dose and high-dose groups were significantly increased after 3 months of administration and it remained in the high level in middle-dose and high-dose group even after 2 weeks of recovery. No drug-related lesions were observed in the histological changes of major organs. Conclusion: The results show that long term use of mulberry concentrated sea-buckthorn beverage can lead to increased CREA, which suggested kidney toxicity. Although no obvious pathological change was found in kidney, we should pay attention to chronic kidney damage in the further research.

\footnotetext{
${ }^{*}$ Corresponding author.
} 


\title{
Keywords
}

\author{
Chinese Traditional Medicine, Chronic Toxicity Test, Mulberry Sea-Buckthorn Beverage \\ Concentrate, Rats
}

\section{Introduction}

In China, traditional Chinese medicines were used in almost all diseases for Chinese patients. Many Chinese people choose traditional Chinese medicines as their favorite because the efficacies of traditional Chinese medicines have been proved by Chinese people's experiences in the past 5000 years. However, the toxicities, especially the chronic toxicities, of traditional Chinese medicines were not comprehensively studied due to the technical limitation of toxicology. In fact, toxicities of some traditional Chinese medicines were reported recently [1] [2]. Safety re-evaluation of traditional Chinese medicines is needed.

Mulberry sea-buckthorn beverage concentrate is composed of the fruit of mulberry and sea-buckthorn. The fruit of mulberry was usually taken as food or medicine in China. Studies showed that the fruit of mulberry contained polyphenol, volatile oil, polysaccharide, amino acid, and so on. To Chinese experiences, mulberry has the efficacy of enhancing immunity, antioxidant, antibacterial, reducing blood sugar, protecting liver and cardiovascular system [3]. Literature [3] has summarized the clinical application of the fruit of mulberry. It can be used for chronic hepatitis, hypertension, hyperlipidemia, diabetes mellitus, aplastic anemia, gastrointestinal diseases (gastric ulcer, duodenal ulcer, chronic gastritis, colitis), senile constipation and sleep disorders, blood deficiency caused by dizziness and tinnitus, sequelae of cerebral concussion, eye disease, et al. [4].

Sea-buckthorn (Hippophae rhamnoides Linn) is one of common deciduous shrubs of Elaeagnaceae; its main chemical constituents are flavonoids, terpenoids, steroids, volatile oil, organic acids, phenols, carbohydrates, including 14 kinds of vitamins, 12 kinds of organic acids and sugar, 22 kinds of amino acid and amide class, 7 kinds of fatty acid, 32 kinds of fat, 30 kinds of phenolic and flavonoid, and 29 kinds of trace elements [5].

Sea-buckthorn has many pharmacological effects and clinical applications, including cardiovascular and liver protection, enhancing immunity, restraining cancer, preventing atherosclerosis and delaying aging [6]. Studies have shown that sea-buckthorn could delay aging in drosophila [6].

Chronic toxicity test, as the main content of pre-clinical toxicity evaluation, has been widely used for preclinical toxicity assessment in medicine and health food. In this study, a chronic toxicity test has been performed to evaluate the chronic toxicity of mulberry sea-buckthorn beverage concentrate.

\section{Materials and Methods}

\subsection{Animals}

120 healthy SPF SD rats, 5 - 6 weeks of age, provided by medical laboratory animal center of Guangdong, were randomly divided into 4 groups with half male and half female, which are control group, low-dose group, middle-dose group and high-dose group. During administration, external signs, behavioral, mental state, reaction, activity, stool, eyes and cavity secretion, hair color, the general condition were daily observed and recorded. And body weight and food intake were measured and recorded weekly. This study has been permitted by Ehics Committee of GDIFDC.

\subsection{Sample and Experimental Design}

Mulberry sea-buckthorn beverage concentrate was produced by Sichuan Chengdu Hongyi Food Co., Ltd., which clinical dose is $0.667 \mathrm{~mL} / \mathrm{kg} \mathrm{BW}$. In this study, the clinical doses of 7.5, 15, 30 times respectively as low-, medium-, high-dose group experiment.

Rats were oral exposed to sample with dosage of $5 \mathrm{~mL} / \mathrm{kg} \mathrm{BW}, 10 \mathrm{~mL} / \mathrm{kg} \mathrm{BW}, 20 \mathrm{~mL} / \mathrm{kg}$ BW by once a day, 6 days a week, 3 months (Weeks 0-13). Negative control group were given equal volume of purified water. 3 months after the last feeding sample, 2/3 rats (half male and half female) were sacrificed for hematological examination, blood biochemistry determination and histopathology analyzing. After additional 2 week (Weeks 14-15) recovery observation, the remaining $1 / 3$ rats were sacrificed and analyzed in the same way. 


\subsection{Hematological Examination}

The collected blood samples were analyzed by automatic blood cell analyzer (BECKMAN COULTER AC.T 5 Diff). Some items were selected for this study, they were red blood cell count (RBC), hemoglobin (HGB), hematocrit (HCT), white blood cell count (WBC), platelet count (PLT), mean red cell volume (MCV), hemoglobin concentration (MCH), mean corpuscular hemoglobin concentration (MCHC), red blood cell volume distribution (RDW), mean platelet volume (MPV) and platelet hematocrit (PCT). Prothrombintime (PT) was measured by Platelet aggregation coagulation analyzer (LG-PABER-I).

\subsection{Blood Biochemistry Determination}

Automatic biochemical analyzer (Hitachi 7020) was used for blood sample analyzing of serum total protein (TP), the serum albumin (ALB), total bilirubin (TBiL), alanine aminotransferase (ALT), aspartate aminotransferase (AST), alkaline phosphatase (ALP), blood glucose (GLU), blood urea nitrogen (BUN), creatinine (CREA), total cholesterol (CHO) and triglyceride (TG).

\subsection{Organ Coefficient}

Heart, liver, spleen, lung, kidney, brain, adrenal, thymus, bladder, the male rat testis, epididymis, female rats ovary, uterus were weighted, and the organ coefficient were calculated according to the following formula.

Organ coefficient $(\%)=$ organ weight $(\mathrm{g}) /$ body weight $(\mathrm{g}) \times 100$.

\subsection{Histological Analysis}

For histopathology, heart, liver, spleen, lung, kidney, stomach, brain, adrenal gland, thyroid gland, bladder, pancreas, trachea, male rats' prostate, testis, epididymis and female rats' ovary, uterus samples were harvested and fixed in $10 \%$ neutral buffered formalin. Subsequently, they were dehydrated in a series of ethanol and embedded in paraffin. Approximately $3.5 \mu \mathrm{m}$ thick sections were cut, mounted on glass slides and stained with hematoxylin and eosin (H\&E). The sections were observed under an optical microscope (ZEISS Axio Scope A1) at different magnifications.

\subsection{Statistical Analysis}

Results were statistically analyzed using SPSS17.0 software. Body and organ weights, food intake, biochemical analysis, Hematological examination and Organ coefficient results were analyzed with a one-way ANOVA with a Bonferroni post-test. For all statistical results, a P-value of $\leq 0.05$ was considered significant.

\section{Results}

\subsection{Influence on the General Condition and Body Weight of Rats}

In this study, all rats showed no obvious abnormalities in animal appearance, behavior, eating, drinking, and all rats were weight increased weekly. Compared with the negative control group, the body-weight and food intake of each group rats showed no significant difference $(\mathrm{P}>0.05)$ in each week through this study (Tables 1-3).

\subsection{Influence on the Blood Cells of Rats}

The influences of mulberry sea-buckthorn beverage concentrate on the blood cells were showed in Table 4. As shown in Table 4, compared with the negative control group, HGB was significantly increased in rats of low-dose group and high-dose group after 3 months oral exposed to mulberry sea-buckthorn beverage concentrate. However, it decreased to normal level after 2 weeks recovery by stopping feed mulberry sea-buckthorn beverage concentrate. The other indexes of Hematological examination showed no significant difference between groups of sample and group of negative control.

\subsection{Influence on Blood Biochemistry of Rats}

The influences of mulberry sea-buckthorn beverage concentrate on the blood biochemistry were showed in 
Table 5. As shown in Table 5, compared with the negative control group, CREA was significantly increased in all sample groups' rats after 3 months oral exposed to mulberry sea-buckthorn beverage concentrate. Furthermore, it still showed significantly increased in rats of middle-dose group and high-dose group after 2 weeks recovery by stopping feed mulberry sea-buckthorn beverage concentrate. The other indexes of blood biochemistry examination showed no significant difference between groups of sample and group of negative control.

Table 1. Influence on the food intake of rats $(\bar{x} \pm s)$.

\begin{tabular}{cccccc}
\hline & Groups & Negative control & Low-dose group & Middle-dose group & High-dose group \\
\hline & Week 1 & $616.33 \pm 34.78$ & $595.72 \pm 41.17$ & $609.03 \pm 20.47$ & $600.82 \pm 24.77$ \\
& Week 2 & $692.25 \pm 81.17$ & $700.02 \pm 68.31$ & $669.87 \pm 57.42$ & $694.53 \pm 46.47$ \\
& Week 3 & $722.63 \pm 107.20$ & $749.17 \pm 85.94$ & $720.73 \pm 71.07$ & $723.07 \pm 80.77$ \\
& Week 4 & $751.57 \pm 112.02$ & $763.18 \pm 101.20$ & $764.62 \pm 102.72$ & $754.87 \pm 94.06$ \\
& Week 5 & $765.13 \pm 97.83$ & $769.35 \pm 121.34$ & $747.17 \pm 99.32$ & $735.28 \pm 90.61$ \\
Food & Week 6 & $767.97 \pm 108.34$ & $798.05 \pm 112.22$ & $770.48 \pm 104.44$ & $765.85 \pm 87.71$ \\
(g/cage) & Week 7 & $753.10 \pm 108.41$ & $741.13 \pm 103.26$ & $749.15 \pm 100.48$ & $709.58 \pm 92.29$ \\
& Week 8 & $721.03 \pm 105.25$ & $715.47 \pm 101.12$ & $701.35 \pm 98.57$ & $671.77 \pm 73.83$ \\
& Week 9 & $701.52 \pm 91.93$ & $719.85 \pm 109.35$ & $697.88 \pm 96.60$ & $682.92 \pm 89.50$ \\
& Week 10 & $729.42 \pm 114.22$ & $748.77 \pm 110.58$ & $716.50 \pm 98.92$ & $712.23 \pm 91.74$ \\
& Week 11 & $728.02 \pm 123.92$ & $756.53 \pm 108.69$ & $729.73 \pm 84.15$ & $719.18 \pm 96.16$ \\
& Week 12 & $720.95 \pm 91.67$ & $755.43 \pm 76.40$ & $699.02 \pm 95.82$ & $711.18 \pm 66.78$ \\
& Week 13 & $525.58 \pm 60.49$ & $524.58 \pm 79.95$ & $507.90 \pm 70.40$ & $499.98 \pm 59.18$ \\
& Week 14 & $722.85 \pm 150.83$ & $748.05 \pm 161.29$ & $740.99 \pm 133.48$ & $732.20 \pm 115.54$ \\
& Week 15 & $722.80 \pm 150.61$ & $778.30 \pm 146.23$ & $776.28 \pm 134.31$ & $773.75 \pm 89.73$ \\
\hline
\end{tabular}

For Weeks 1-13, n = 6 cages, 5 rats/cage. For Weeks 14-15, n = 2 cages, 5 rats/cage.

Table 2. Influence on body-weight of female rats $(\bar{x} \pm s)$.

\begin{tabular}{|c|c|c|c|c|c|}
\hline & Groups & Negative control & Low-dose group & Middle-dose group & High-dose group \\
\hline \multirow{16}{*}{$\begin{array}{c}\text { Body } \\
\text { weight } \\
\text { (g) }\end{array}$} & Week 0 & $91.06 \pm 3.80$ & $90.94 \pm 3.77$ & $89.42 \pm 4.55$ & $89.94 \pm 3.08$ \\
\hline & Week 1 & $124.66 \pm 5.47$ & $125.49 \pm 6.97$ & $126.12 \pm 5.46$ & $125.39 \pm 4.30$ \\
\hline & Week 2 & $150.23 \pm 6.96$ & $153.06 \pm 8.46$ & $151.85 \pm 7.17$ & $151.54 \pm 4.17$ \\
\hline & Week 3 & $173.31 \pm 9.85$ & $177.64 \pm 11.58$ & $180.70 \pm 10.56$ & $177.78 \pm 6.95$ \\
\hline & Week 4 & $188.19 \pm 11.76$ & $195.07 \pm 13.92$ & $195.60 \pm 10.34$ & $195.83 \pm 7.73$ \\
\hline & Week 5 & $202.90 \pm 11.98$ & $207.67 \pm 14.67$ & $208.45 \pm 10.56$ & $207.51 \pm 7.11$ \\
\hline & Week 6 & $216.22 \pm 13.43$ & $218.99 \pm 14.92$ & $219.35 \pm 11.56$ & $218.59 \pm 8.04$ \\
\hline & Week 7 & $224.07 \pm 14.72$ & $226.62 \pm 16.16$ & $227.39 \pm 12.50$ & $224.06 \pm 8.21$ \\
\hline & Week 8 & $230.61 \pm 15.84$ & $237.75 \pm 16.08$ & $234.26 \pm 14.22$ & $233.74 \pm 10.87$ \\
\hline & Week 9 & $239.44 \pm 16.58$ & $243.67 \pm 17.35$ & $243.12 \pm 13.08$ & $240.33 \pm 10.01$ \\
\hline & Week 10 & $246.29 \pm 19.12$ & $249.82 \pm 17.77$ & $249.01 \pm 15.31$ & $245.57 \pm 10.15$ \\
\hline & Week 11 & $251.25 \pm 18.93$ & $255.11 \pm 18.45$ & $254.36 \pm 14.28$ & $251.94 \pm 13.14$ \\
\hline & Week 12 & $256.09 \pm 19.28$ & $266.21 \pm 20.56$ & $258.29 \pm 15.68$ & $259.42 \pm 15.12$ \\
\hline & Week 13 & $262.86 \pm 20.04$ & $264.6 \pm 20.70$ & $260.41 \pm 16.04$ & $258.63 \pm 15.04$ \\
\hline & Week 14 & $263.43 \pm 31.77$ & $281.43 \pm 21.80$ & $274.85 \pm 15.66$ & $268.33 \pm 8.38$ \\
\hline & Week 15 & $266.73 \pm 24.21$ & $279.33 \pm 24.00$ & $278.15 \pm 9.75$ & $270.38 \pm 8.58$ \\
\hline
\end{tabular}

For Weeks 0-13, n=14. For Weeks 14-15, n= 4 . 
Table 3. Influence on body-weight of male rats $(\bar{x} \pm s)$.

\begin{tabular}{cccccc}
\hline & Groups & Negative control & Low-dose group & Middle-dose group & High-dose group \\
\hline & Week 0 & $98.52 \pm 3.64$ & $98.50 \pm 4.60$ & $98.29 \pm 4.97$ & $96.56 \pm 6.76$ \\
& Week 1 & $149.91 \pm 9.53$ & $147.01 \pm 5.79$ & $146.66 \pm 6.72$ & $146.54 \pm 9.25$ \\
& Week 2 & $196.74 \pm 10.90$ & $196.84 \pm 7.77$ & $193.98 \pm 10.26$ & $192.09 \pm 10.34$ \\
& Week 3 & $240.77 \pm 11.23$ & $241.71 \pm 11.05$ & $239.99 \pm 10.93$ & $237.49 \pm 10.15$ \\
& Week 4 & $278.31 \pm 13.98$ & $279.09 \pm 16.39$ & $278.61 \pm 16.12$ & $276.55 \pm 10.76$ \\
Body & Week 5 & $310.09 \pm 14.71$ & $313.66 \pm 14.49$ & $312.64 \pm 15.99$ & $306.60 \pm 13.73$ \\
(g) & Week 6 & $333.54 \pm 15.96$ & $337.06 \pm 19.34$ & $332.01 \pm 20.41$ & $328.91 \pm 14.88$ \\
& Week 7 & $352.72 \pm 17.71$ & $358.74 \pm 16.02$ & $355.88 \pm 21.34$ & $340.98 \pm 18.70$ \\
& Week 8 & $364.72 \pm 17.78$ & $369.91 \pm 19.14$ & $368.01 \pm 27.26$ & $359.38 \pm 21.69$ \\
& Week 9 & $379.83 \pm 21.00$ & $385.04 \pm 22.07$ & $383.04 \pm 29.74$ & $372.39 \pm 23.65$ \\
& Week 10 & $394.84 \pm 25.37$ & $400.12 \pm 21.73$ & $394.70 \pm 33.86$ & $386.28 \pm 24.75$ \\
& Week 11 & $406.89 \pm 27.73$ & $415.04 \pm 21.18$ & $405.58 \pm 34.11$ & $399.01 \pm 26.77$ \\
& Week 12 & $409.27 \pm 32.89$ & $423.30 \pm 19.97$ & $414.18 \pm 32.36$ & $408.64 \pm 27.11$ \\
& Week 13 & $419.31 \pm 28.47$ & $431.71 \pm 19.01$ & $416.99 \pm 38.48$ & $410.37 \pm 27.24$ \\
\hline
\end{tabular}

For Weeks 0-13, $n=14$. For Weeks 14-15, $n=4$.

Table 4. Influence on the blood cells of rats $(\bar{x} \pm s)$.

\begin{tabular}{|c|c|c|c|c|c|}
\hline & Groups & Negative control & Low-dose group & Middle-dose group & High-dose group \\
\hline \multirow{12}{*}{$\begin{array}{l}\text { After feeding } \\
\text { samples for } 3 \\
\text { months } \\
(\mathrm{n}=16)\end{array}$} & PT (s) & $13.60 \pm 0.43$ & $13.30 \pm 0.45$ & $13.35 \pm 0.54$ & $13.34 \pm 0.39$ \\
\hline & WBC $\left(\times 10^{9} / \mathrm{L}\right)$ & $6.14 \pm 1.23$ & $6.42 \pm 1.30$ & $6.30 \pm 1.91$ & $6.27 \pm 1.48$ \\
\hline & $\mathrm{RBC}\left(\times 10^{12} / \mathrm{L}\right)$ & $8.618 \pm 0.439$ & $8.909 \pm 0.431$ & $8.818 \pm 0.578$ & $8.887 \pm 0.456$ \\
\hline & HGB (g/dL) & $16.48 \pm 0.60$ & $17.12 \pm 0.69^{* *}$ & $16.69 \pm 0.87$ & $17.28 \pm 0.66^{* *}$ \\
\hline & HCT (\%) & $47.69 \pm 2.08$ & $48.86 \pm 1.97$ & $47.71 \pm 2.44$ & $48.79 \pm 2.28$ \\
\hline & MCV (fL) & $54.3 \pm 1.1$ & $54.6 \pm 1.3$ & $54.3 \pm 1.4$ & $54.8 \pm 1.1$ \\
\hline & MCH (pg) & $18.92 \pm 0.54$ & $19.18 \pm 0.60$ & $18.86 \pm 0.59$ & $19.09 \pm 0.55$ \\
\hline & MCHC (g/dL) & $34.87 \pm 0.39$ & $35.03 \pm 0.49$ & $34.95 \pm 0.29$ & $34.91 \pm 0.38$ \\
\hline & RDW (\%) & $11.44 \pm 0.40$ & $11.45 \pm 0.32$ & $11.44 \pm 0.54$ & $11.54 \pm 0.32$ \\
\hline & $\operatorname{PLT}\left(\times 10^{9} / \mathrm{L}\right)$ & $866.5 \pm 88.5$ & $882.6 \pm 128.4$ & $920.2 \pm 61.4$ & $910.1 \pm 62.8$ \\
\hline & MPV (fL) & $7.44 \pm 0.37$ & $7.67 \pm 0.51$ & $7.44 \pm 0.23$ & $7.44 \pm 0.37$ \\
\hline & PCT (\%) & $0.6362 \pm 0.0730$ & $0.6543 \pm 0.1015$ & $0.6681 \pm 0.0583$ & $0.6652 \pm 0.0550$ \\
\hline \multirow{12}{*}{$\begin{array}{l}\text { After recovery } \\
\text { for } 2 \text { weeks } \\
\quad(\mathrm{n}=8)\end{array}$} & PT (s) & $13.81 \pm 0.41$ & $13.38 \pm 0.53$ & $13.79 \pm 0.45$ & $13.79 \pm 0.37$ \\
\hline & WBC $\left(\times 10^{9} / \mathrm{L}\right)$ & $6.00 \pm 2.11$ & $5.50 \pm 0.94$ & $5.74 \pm 1.91$ & $6.35 \pm 1.14$ \\
\hline & $\mathrm{RBC}\left(\times 10^{12} / \mathrm{L}\right)$ & $8.754 \pm 0.657$ & $8.904 \pm 0.309$ & $8.975 \pm 0.625$ & $9.074 \pm 0.304$ \\
\hline & HGB (g/dL) & $17.08 \pm 1.14$ & $17.05 \pm 0.60$ & $16.95 \pm 0.75$ & $17.21 \pm 0.37$ \\
\hline & HCT (\%) & $47.91 \pm 2.98$ & $47.63 \pm 1.71$ & $47.88 \pm 2.49$ & $48.41 \pm 0.66$ \\
\hline & MCV (fL) & $54.4 \pm 1.4$ & $53.1 \pm 1.1$ & $53.8 \pm 1.0$ & $53.8 \pm 1.0$ \\
\hline & MCH (pg) & $19.45 \pm 0.55$ & $18.94 \pm 0.53$ & $19.13 \pm 0.49$ & $19.04 \pm 0.46$ \\
\hline & MCHC (g/dL) & $35.73 \pm 0.21$ & $35.61 \pm 0.20$ & $35.45 \pm 0.43$ & $35.58 \pm 0.18$ \\
\hline & RDW (\%) & $10.84 \pm 0.37$ & $11.15 \pm 0.26$ & $11.10 \pm 0.54$ & $11.05 \pm 0.40$ \\
\hline & $\operatorname{PLT}\left(\times 10^{9} / \mathrm{L}\right)$ & $874.1 \pm 79.5$ & $844.1 \pm 42.2$ & $904.3 \pm 103.2$ & $881.6 \pm 94.9$ \\
\hline & MPV (fL) & $7.65 \pm 0.29$ & $7.43 \pm 0.19$ & $7.39 \pm 0.36$ & $7.61 \pm 0.33$ \\
\hline & PCT (\%) & $0.6655 \pm 0.0554$ & $0.6226 \pm 0.0356$ & $0.6683 \pm 0.0988$ & $0.6583 \pm 0.0782$ \\
\hline
\end{tabular}

\footnotetext{
${ }^{* *}$ Represent for $\mathrm{P}<0.01$.
} 
Table 5. Influence on blood biochemistry of rats $(\bar{x} \pm \mathrm{s})$.

\begin{tabular}{|c|c|c|c|c|c|}
\hline & Groups & Negative control & Low-dose group & Middle-dose group & High-dose group \\
\hline \multirow{11}{*}{$\begin{array}{l}\text { After feeding } \\
\text { samples for } 3 \\
\text { months } \\
(\mathrm{n}=16)\end{array}$} & ALT (U/L) & $41.9 \pm 5.8$ & $45.0 \pm 8.0$ & $41.6 \pm 6.0$ & $43.9 \pm 7.7$ \\
\hline & AST (U/L) & $100.3 \pm 8.1$ & $106.3 \pm 10.5$ & $101.2 \pm 11.3$ & $103.1 \pm 10.3$ \\
\hline & $\operatorname{ALP}(\mathrm{U} / \mathrm{L})$ & $101.1 \pm 18.0$ & $105.8 \pm 22.9$ & $101.4 \pm 16.9$ & $95.4 \pm 13.4$ \\
\hline & TbiL (mmol/L) & $1.781 \pm 0.811$ & $2.007 \pm 0.844$ & $2.036 \pm 0.966$ & $1.783 \pm 1.252$ \\
\hline & BUN (mmol/L) & $5.786 \pm 0.381$ & $6.024 \pm 0.409$ & $5.854 \pm 0.492$ & $6.041 \pm 0.437$ \\
\hline & CREA (mmol/L) & $26.08 \pm 1.70$ & $28.79 \pm 3.14^{* *}$ & $30.80 \pm 3.21^{* *}$ & $30.80 \pm 3.79^{* *}$ \\
\hline & GLU (mmol/L) & $8.228 \pm 0.614$ & $8.087 \pm 0.407$ & $8.012 \pm 0.610$ & $7.775 \pm 0.771$ \\
\hline & TP (g/L) & $62.02 \pm 1.97$ & $62.46 \pm 2.02$ & $61.00 \pm 2.53$ & $60.65 \pm 2.46$ \\
\hline & $\operatorname{ALB}(g / L)$ & $33.10 \pm 2.30$ & $33.05 \pm 2.16$ & $32.85 \pm 2.51$ & $32.31 \pm 1.80$ \\
\hline & $\mathrm{TG}(\mathrm{mmol} / \mathrm{L})$ & $0.540 \pm 0.100$ & $0.567 \pm 0.095$ & $0.539 \pm 0.125$ & $0.555 \pm 0.068$ \\
\hline & CHOL (mmol/L) & $1.805 \pm 0.276$ & $1.906 \pm 0.220$ & $1.977 \pm 0.210$ & $1.821 \pm 0.166$ \\
\hline \multirow{11}{*}{$\begin{array}{l}\text { After recovery } \\
\text { for } 2 \text { weeks } \\
\quad(\mathrm{n}=8)\end{array}$} & $\operatorname{ALT}(\mathrm{U} / \mathrm{L})$ & $36.0 \pm 5.7$ & $36.1 \pm 6.3$ & $37.4 \pm 5.9$ & $35.0 \pm 4.7$ \\
\hline & AST (U/L) & $106.6 \pm 6.2$ & $109.9 \pm 7.2$ & $108.3 \pm 11.9$ & $107.0 \pm 3.6$ \\
\hline & ALP (U/L) & $86.0 \pm 12.2$ & $80.0 \pm 18.7$ & $77.4 \pm 20.3$ & $78.6 \pm 14.6$ \\
\hline & TbiL (mmol/L) & $1.530 \pm 0.989$ & $2.058 \pm 0.652$ & $1.976 \pm 0.833$ & $1.515 \pm 1.089$ \\
\hline & BUN (mmol/L) & $5.956 \pm 0.563$ & $6.015 \pm 0.461$ & $6.260 \pm 0.548$ & $6.334 \pm 0.320$ \\
\hline & CREA (mmol/L) & $30.10 \pm 4.54$ & $31.68 \pm 4.22$ & $37.33 \pm 4.57^{* *}$ & $37.01 \pm 3.50^{* *}$ \\
\hline & GLU (mmol/L) & $8.111 \pm 0.332$ & $7.968 \pm 0.461$ & $8.445 \pm 0.801$ & $8.166 \pm 0.778$ \\
\hline & TP (g/L) & $59.90 \pm 3.14$ & $60.59 \pm 4.46$ & $60.03 \pm 2.69$ & $59.20 \pm 3.37$ \\
\hline & $\operatorname{ALB}(g / L)$ & $32.91 \pm 1.80$ & $33.09 \pm 2.30$ & $33.00 \pm 1.62$ & $32.19 \pm 1.61$ \\
\hline & $\mathrm{TG}(\mathrm{mmol} / \mathrm{L})$ & $0.514 \pm 0.072$ & $0.535 \pm 0.056$ & $0.535 \pm 0.092$ & $0.599 \pm 0.106$ \\
\hline & CHOL (mmol/L) & $1.879 \pm 0.331$ & $2.001 \pm 0.290$ & $1.973 \pm 0.169$ & $1.840 \pm 0.130$ \\
\hline
\end{tabular}

${ }^{* *}$ Represent for $\mathrm{P}<0.01$.

\subsection{Influence on Organ Coefficient of Rats}

In this study, all rats showed no obvious abnormalities in organ size and weight. Results were showed in Table 6. Compared with the negative control group, the organ coefficient of each group rats showed no significant difference $(\mathrm{P}>0.05)$.

\subsection{Histological Analysis}

After administration for 3 months and after recovery for 2 weeks, major organs of rats were picked and carefully examined when anatomizing, which were heart, liver, spleen, lung, kidney, stomach, brain, adrenal gland, thyroid gland, pancreatic, bladder, trachea, thymus, male rats' prostate, testis, seminal vesicle and female rats' ovary, uterus. And no pathological changes were seen. Some major organs were selected for histological analysis. And histopathological assessment of the heart, liver, spleen, lung, kidney, stomach, brain, adrenal, thyroid, pancreas, trachea, bladder, prostate, male rats' testis, epididymis and female rats' ovary, uterus showed no differences between the treated animals and the controls (data no shown).

\section{Discussion}

Compared with the negative control group, HGB significantly increased in rats of low-dose group and high-dose group after 3 months oral exposed to mulberry sea-buckthorn beverage concentrate. However, it decreased to normal level after 2 weeks recovery by stopping feed mulberry sea-buckthorn beverage concentrate. Hemoglobin is a pigment containing cofactor binding protein, synthetized by the human nucleated red blood cells and reticulocytes, mainly in the organism responsible for carrying oxygen. 
Table 6. Influence on organ coefficient of rats $(\bar{x} \pm s)$.

\begin{tabular}{|c|c|c|c|c|c|}
\hline & Groups & Negative control & Low-dose group & Middle-dose group & High-dose group \\
\hline \multirow{13}{*}{$\begin{array}{l}\text { Organ coefficient } \\
\text { of rats after } \\
\text { feeding samples } \\
\text { for } 3 \text { months (\%) } \\
\quad(n=16)\end{array}$} & Brain & $0.5984 \pm 0.1331$ & $0.5988 \pm 0.1286$ & $0.6060 \pm 0.1311$ & $0.6209 \pm 0.1234$ \\
\hline & Liver & $2.4979 \pm 0.1527$ & $2.5800 \pm 0.1665$ & $2.5275 \pm 0.1432$ & $2.5821 \pm 0.1434$ \\
\hline & Kidney & $0.6577 \pm 0.0329$ & $0.6545 \pm 0.0527$ & $0.6671 \pm 0.0340$ & $0.6801 \pm 0.0423$ \\
\hline & Spleen & $0.1598 \pm 0.0137$ & $0.1537 \pm 0.0180$ & $0.1597 \pm 0.0207$ & $0.1584 \pm 0.0162$ \\
\hline & Lung & $0.3866 \pm 0.0537$ & $0.3899 \pm 0.0511$ & $0.3974 \pm 0.0600$ & $0.4079 \pm 0.0541$ \\
\hline & Heart & $0.3460 \pm 0.0346$ & $0.3306 \pm 0.0321$ & $0.3400 \pm 0.0438$ & $0.3529 \pm 0.0410$ \\
\hline & Thymus & $0.1027 \pm 0.0311$ & $0.1164 \pm 0.0359$ & $0.1057 \pm 0.0259$ & $0.0996 \pm 0.0199$ \\
\hline & Adrenal gland & $0.0191 \pm 0.0077$ & $0.0198 \pm 0.0068$ & $0.0220 \pm 0.0073$ & $0.0202 \pm 0.0065$ \\
\hline & Testis & $0.8150 \pm 0.0357$ & $0.8044 \pm 0.0590$ & $0.8513 \pm 0.1002$ & $0.8447 \pm 0.0477$ \\
\hline & Epididymis & $0.3043 \pm 0.0240$ & $0.3027 \pm 0.0445$ & $0.3147 \pm 0.0377$ & $0.3091 \pm 0.0114$ \\
\hline & Ovary & $0.0402 \pm 0.0101$ & $0.0409 \pm 0.0076$ & $0.0447 \pm 0.0062$ & $0.0406 \pm 0.0108$ \\
\hline & Uterus & $0.2827 \pm 0.0774$ & $0.2451 \pm 0.0502$ & $0.2700 \pm 0.0832$ & $0.2386 \pm 0.0456$ \\
\hline & Bladder & $0.0288 \pm 0.0066$ & $0.0302 \pm 0.0050$ & $0.0289 \pm 0.0051$ & $0.0310 \pm 0.0040$ \\
\hline \multirow{13}{*}{$\begin{array}{l}\text { Organ coefficient } \\
\text { of rats after } \\
\text { recovery for } 2 \\
\text { weeks }(\%) \\
(\mathrm{n}=8)\end{array}$} & Brain & $0.6003 \pm 0.1025$ & $0.5741 \pm 0.1211$ & $0.5819 \pm 0.1330$ & $0.6156 \pm 0.1434$ \\
\hline & Liver & $2.5161 \pm 0.1186$ & $2.4622 \pm 0.0764$ & $2.4597 \pm 0.0999$ & $2.5195 \pm 0.0689$ \\
\hline & Kidney & $0.6317 \pm 0.0343$ & $0.6469 \pm 0.0343$ & $0.6415 \pm 0.0271$ & $0.6586 \pm 0.0401$ \\
\hline & Spleen & $0.1396 \pm 0.0060$ & $0.1459 \pm 0.0118$ & $0.1355 \pm 0.0059$ & $0.1480 \pm 0.0074$ \\
\hline & Lung & $0.3815 \pm 0.0258$ & $0.3865 \pm 0.0463$ & $0.3680 \pm 0.0517$ & $0.4058 \pm 0.0447$ \\
\hline & Heart & $0.3314 \pm 0.0343$ & $0.3540 \pm 0.0460$ & $0.3332 \pm 0.0326$ & $0.3464 \pm 0.0570$ \\
\hline & Thymus & $0.0917 \pm 0.0113$ & $0.0936 \pm 0.0171$ & $0.0958 \pm 0.0199$ & $0.0998 \pm 0.0279$ \\
\hline & Adrenal gland & $0.0182 \pm 0.0036$ & $0.0184 \pm 0.0053$ & $0.0180 \pm 0.0058$ & $0.0192 \pm 0.0062$ \\
\hline & Testis & $0.7738 \pm 0.0997$ & $0.7580 \pm 0.0255$ & $0.7779 \pm 0.0931$ & $0.7575 \pm 0.0101$ \\
\hline & Epididymis & $0.2724 \pm 0.0328$ & $0.2703 \pm 0.0393$ & $0.2698 \pm 0.0216$ & $0.2657 \pm 0.0186$ \\
\hline & Ovary & $0.0280 \pm 0.0039$ & $0.0315 \pm 0.0026$ & $0.0312 \pm 0.0008$ & $0.0363 \pm 0.0057$ \\
\hline & Uterus & $0.2324 \pm 0.0715$ & $0.2129 \pm 0.0166$ & $0.2498 \pm 0.0801$ & $0.2731 \pm 0.0508$ \\
\hline & Bladder & $0.0284 \pm 0.0058$ & $0.0265 \pm 0.0087$ & $0.0263 \pm 0.0054$ & $0.0290 \pm 0.0061$ \\
\hline
\end{tabular}

Note: For uterus, ovary, testis and epididymis, $\mathrm{n}=8$ for rats after feeding samples for 3 months; $\mathrm{n}=4$ for rats after recovery for 2 weeks.

In clinic, the HGB value increased probably due to water loss in the plasma, blood concentration, such as series fierce vomiting, severe diarrhea, chronic adrenocortical hypofunction, diabetes insipidus, hyperthyroidism and other diseases. The increased HGB value can also cause hypoxia. Hyposia leads to the increase of serum erythropoietin, and subsequently stimulates the release of red cells from bone marrow.

Research has shown that sea-buckthorn can obviously improve the athlete's vital capacity, hemoglobin, and cardiac function [7]. Another research shows that 30\% mulberry liquid, once daily by gavage of $1 \mathrm{~mL}$ for 5 days can recover the HGB value of rats, which have been injected by acetyl phenylhydrazine to decrease the HGB value, to normal level [8].

In this study, the significant increase of HGB value appears in the low-dose group and high-dose group presumably due to the normal pharmacological effect of mulberry or sea-buckthorn.

Creatinine (CREA) is a small molecular substance. Almost all the daily produced creatinines in vivo are excreted in the urine. Because the value of CREA generally unaffected by urine volume, detection of CREA is one of the main methods used to understand the renal function in clinic. The concentration of CREA was determined mainly by the glomerular filtration rate. The decreased filtration capacity of glomerular leads to the higher concentration of CREA. So, CREA value can reflect the renal parenchyma damage.

In this study, CREA was significantly increased in all sample groups' rats after 3 months oral exposed to mulberry sea-buckthorn beverage concentrate. And the increase amplitude of CREA was positively related to 
the dosage of mulberry sea-buckthorn beverage concentrate. Furthermore, it still showed the significant increase in rats of middle-dose group and high-dose group after 2 weeks recovery by stopping feed mulberry sea-buckthorn beverage concentrate. These results indicated that mulberry sea-buckthorn beverage concentrate could damage the kidney. However, related lesions were not found in the renal histopathological examination, and the organ coefficient of kidney showed no difference between sample groups and negative control group. This indicates that the damage on kidney is not serious.

\section{References}

[1] Efferth, T. and Kaina, B. (2011) Toxicities by Herbal Medicines with Emphasis to Traditional Chinese Medicine. Current Drug Metabolism, 12, 989-996. http://dx.doi.org/10.2174/138920011798062328

[2] Zhou, J., Ouedraogo, M., Qu, F. and Duez, P. (2013) Potential Genotoxicity of Traditional Chinese Medicinal Plants and Phytochemicals: An Overview. Phytotherapy Research, 27, 1745-1755. http://dx.doi.org/10.1002/ptr.4942

[3] Xia, Y.J., Tan, Z.P., Wang, L.P. and Xin, N. (2013) Research Progress of Chinese Herbal Mulberry Used as Medicine or Food. China Medicine and Pharmacy, 3, 52-54.

[4] Chao, H.Y. (1999) Research Progress of Mulberry. Lishizhen Medicine and Materia Medica Research, 10, $6262-6281$.

[5] Ge, X.Y. (1986) Survey of Studies on the Chemical Constituents of Sea-Buckthorn. Chinese Traditional and Herbal Drugs, 17, 42.

[6] Ruan, J.C., Huang, Y.X. and Yang, S.Y. (1995) The Acute Toxicity and Anti-Aging Test of Seabuckthorn. Chinese Journal of New Drugs and Clinical Remedies, 14, 325-327.

[7] Lu, K.P. (1984) Effects of Sea-Buckthorn Essence for Athletic Ability. Inner Mongolia Medical Journal, 2, 8.

[8] Chu, W. and Xu, J. (1994) Effect of Mulberry Fruit on Blood Deficiency, Blood Stasis and Immunity. Hubei Journal of Traditional Chinese Medicine, 16, 46-47. 
Scientific Research Publishing (SCIRP) is one of the largest Open Access journal publishers. It is currently publishing more than 200 open access, online, peer-reviewed journals covering a wide range of academic disciplines. SCIRP serves the worldwide academic communities and contributes to the progress and application of science with its publication.

Other selected journals from SCIRP are listed as below. Submit your manuscript to us via either submit@scirp.org or Online Submission Portal.
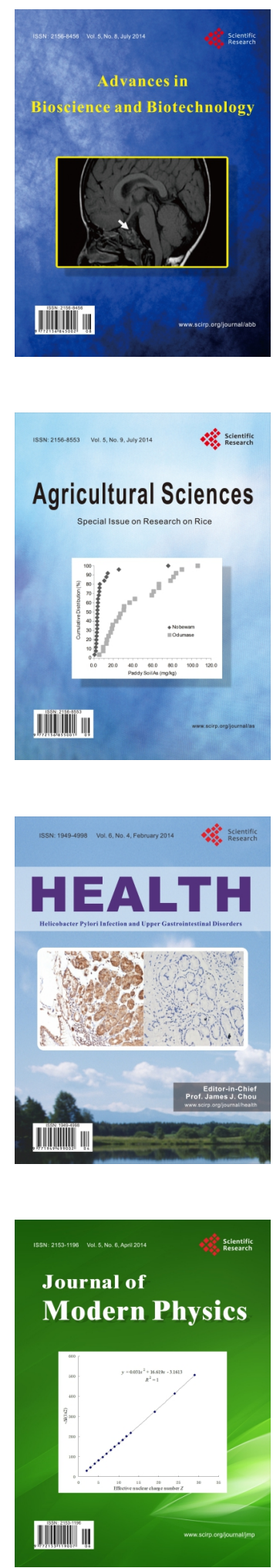
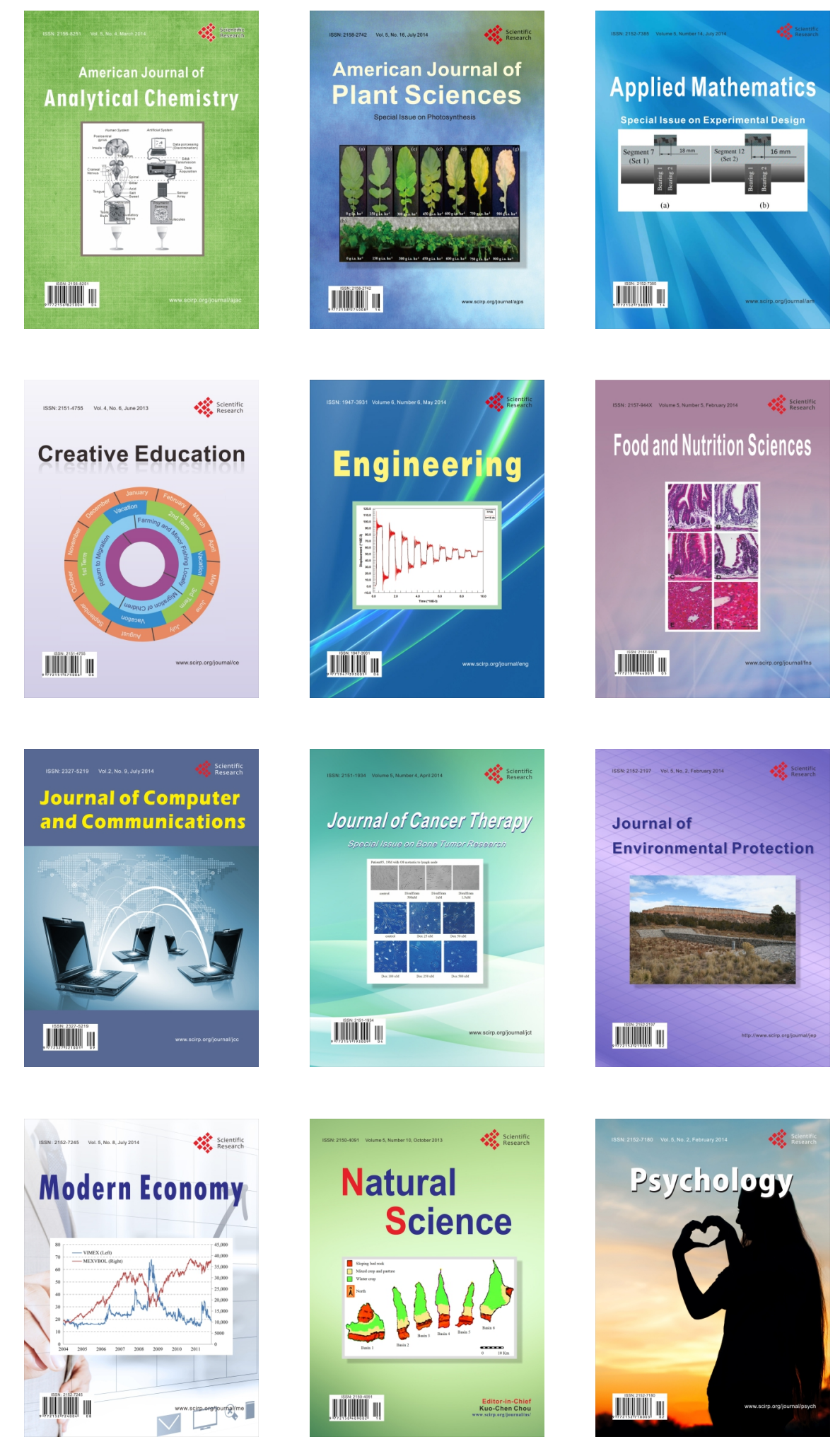